\title{
Lianas de uma floresta estacional semidecidual, Município de Paulo de Faria, Norte do Estado de São Paulo, Brasil ${ }^{1}$
}

\author{
ANDRÉIA ALVES REZENDE 2,5 NEUSA TARODA RANGA ${ }^{3} \mathrm{e}$ \\ RODRIGO AUGUSTO SANTINELO PEREIRA ${ }^{4}$
}

(recebido: 10 de agosto de 2006; aceito: 9 de agosto de 2007)

\begin{abstract}
Lianas of a seasonal semideciduous forest, Paulo de Faria, northern São Paulo State, Brazil). Lianas are soilgerminating plants, perennially rooted that need support to reach the canopy. In tropical forest they are an important form of life due to their diversity, they are part of the forest structure, and source of food for various animals species. The aim of this study was to evaluate the species composition, the diversity and the structure of a liana community in a seasonal semideciduous forest at the Ecological Station of Paulo de Faria, São Paulo, Brazil. The sampling included lianas specimens with DBH $\geq 1 \mathrm{~cm}$ collected in 100 plots of $10 \times 10 \mathrm{~m}$ ( 1 ha). We collected 1,427 individuals representing 45 species. The species diversity $\left(H^{\prime}\right)$ was 2.98 nats individual $^{-1}$. The richest families were Bignoniaceae (14 species), Sapindaceae (nine), Malpighiaceae (six) and Leguminosae (four), similarly to other neotropical forests. The families Bignoniaceae, Sapindaceae and Apocynaceae were represented by a great number of individuals, Melloa quadrivalvis (Bignoniaceae) was the most important species. The majority of the sampled individuals (57.6\%) had diameters between $1-2.5 \mathrm{~cm}$ and in 26 individuals only the diameter was larger than $10 \mathrm{~cm}$. If the purpose of a study of a liana community is to estimate the abundance our data suggest that all individuals with DBH $>1 \mathrm{~cm}$ must be sampled.
\end{abstract}

Key words - abundance, diversity, liana, phytosociological structure

RESUMO - (Lianas de uma floresta estacional semidecidual, Município de Paulo de Faria, Norte do Estado de São Paulo, Brasil). Lianas são plantas que germinam no solo, se mantêm enraizadas durante toda a vida e necessitam de suporte físico para alcançarem o dossel. Nas florestas tropicais, constituem uma forma de vida importante em virtude de sua diversidade, por fazerem parte da estrutura da floresta e, além disso, servirem de alimento para uma variedade de animais. Este trabalho teve como objetivo avaliar a composição de espécies, a diversidade e a estrutura da comunidade de lianas em um fragmento de floresta estacional semidecidual, na Estação Ecológica de Paulo de Faria, São Paulo, Brasil. Amostraram-se os indivíduos de lianas com DAP $\geq 1 \mathrm{~cm}$ em 100 parcelas de 10 x $10 \mathrm{~m}$ (1 ha), resultando em 1.427 indivíduos de 45 espécies, o que representou uma diversidade $\left(H^{\prime}\right)$ de 2,98 nats indivíduo ${ }^{-1}$. As famílias mais ricas em espécies foram Bignoniaceae (14 espécies), Sapindaceae (nove), Malpighiaceae (seis) e Leguminosae (quatro), semelhante ao observado em outras florestas neotropicais. As famílias Bignoniaceae, Sapindaceae e Apocynaceae foram representadas por grande número de indivíduos, sendo Melloa quadrivalvis a espécie com maior valor de importância. A maioria dos indivíduos amostrados $(57,6 \%)$ apresentou diâmetro $<2,5 \mathrm{~cm}$, somente 26 indivíduos tiveram o diâmetro $>10 \mathrm{~cm}$. Nossos dados sugerem que se o objetivo for estimar a abundância, todos os indivíduos com DAP $>1 \mathrm{~cm}$ devem ser amostrados.

Palavras-chave - abundância, diversidade, fitossociologia, liana

\section{Introdução}

A maioria dos estudos sobre ecologia de lianas foi realizada em florestas tropicais (Hegarty \& Caballé

1. Parte da tese de doutorado de A. A. Rezende, Programa de Pós Graduação em Biologia Vegetal da Universidade Estadual de Campinas.

2. Centro Universitário de Rio Preto, Departamento de Ciências Biológicas, Rua Yvette Gabriel Atique, 45, 15025-400 São José do Rio Preto, SP, Brasil.

3. Universidade Estadual Paulista, Instituto de Biociências Letras e Ciências Exatas, Departamento de Zoologia e Botânica, Rua Cristóvão Colombo, 2265, 15054-000 São José do Rio Preto, SP, Brasil.

4. Universidade de São Paulo, Faculdade de Filosofia Ciências e Letras de Ribeirão Preto, Departamento de Biologia, Avenida Bandeirantes, 3900, Setor de Botânica, 14040-901 Ribeirão Preto, SP, Brasil.

5. Autor para correspondência: rezende_bot@yahoo.com.br
1991, Schnitzer \& Bongers 2002, Pérez-Salicrup et al. 2004), atribuindo-se grande importância aos aspectos de abundância, riqueza, diversidade e associação de espécies de árvores e lianas. Estudos mostram que sua abundância é maior em clareiras da floresta, nos vales, nos solos ricos em nutrientes, em florestas com estação seca bem marcada e em florestas de inundação temporal, onde a disponibilidade de suporte utilizada para a sua sustentação é maior (Putz 1984, Gentry 1991, Hegarty \& Caballé 1991, Balfour \& Bond 1993).

Apesar de sua reconhecida importância e ampla distribuição ecológica, a atenção dirigida ao estudo das espécies de lianas tem sido pequena, sendo um dos componentes da estrutura da floresta menos estudado, devido à dificuldade de coleta em florestas densas $\mathrm{e}$ por ocuparem preferencialmente as copas das árvores (Gentry 1991). Os estudos realizados recentemente em 
muitas florestas, como os de Muthuramkumar \& Parthasarathy (2000), Nabe-Nielsen (2001), Perez-Salicrup (2001), Schnitzer \& Bongers (2002), Parthasarathy et al. (2004), Rice et al. (2004), Schnitzer (2005), Gerwing et al. (2006), aumentaram o conhecimento sobre a ecologia de lianas. No Brasil, estudos que tratam da florística deste grupo tem aumentado (Kim 1996, Morellato \& Leitão Filho 1998, Udulutsch et al. 2004, Rezende \& Ranga 2005), mas os que abordam seus aspectos ecológicos nas florestas são raros. Dentre estes, destacam-se trabalhos referentes a fitossociologia (Hora \& Soares 2002), mortalidade e crescimento em diâmetro (Lombardi et al. 1999), fenologia (Morellato \& Leitão Filho 1996), abundância relacionada a diferentes históricos de perturbações (Toniato \& Oliveira-Filho 2004) e sobre manejo em fragmentos florestais (Engel et al. 1998, Rozza 2003), realizados em florestas semidecíduas. Estudos básicos sobre a comunidade de lianas, tais como abundância relativa, associação liana-forófito e referentes às mudanças ao longo do tempo, continuam ainda pouco explorados. Em programas silviculturais, o controle de lianas tem sido recomendado como ferramenta de manejo florestal (Engel et al. 1998). Porém, quando o enfoque é a conservação de fragmentos florestais e não a produção de madeira, os possíveis impactos ecológicos decorrentes da eliminação das lianas não são conhecidos (Johns 1988). Devido à escassez de conhecimento de sua biologia e a sua importância nos fragmentos florestais, muitas questões sobre o manejo e a conservação de florestas ainda estão sem respostas. Um maior número de estudos a respeito deste grupo de plantas poderá preencher esta lacuna no conhecimento da ecologia vegetal.

Este estudo teve como objetivo avaliar a composição, a diversidade, a distribuição de espécies e a estrutura da comunidade de lianas de um trecho de floresta estacional semidecidual, sabendo-se que o conhecimento desses parâmetros é básico para o estudo de comunidades florestais.

\section{Material e métodos}

Área de estudo - Este estudo foi realizado na Estação Ecológica de Paulo de Faria (EEPF), localizada ao norte do Estado de São Paulo, Brasil (19 $55^{\circ}-19^{\circ} 58^{\prime}$ S e $49^{\circ} 31^{\prime}-49^{\circ} 32^{\prime}$ W), às margens do Rio Grande, na divisa com o Estado de Minas Gerais. A área de floresta encontra-se em altitude que varia de 400 a $495 \mathrm{~m}$ e abrange cerca de 650 ha, sendo que 435,73 ha correspondem à EEPF. Na região, o clima é do tipo Aw, tropical quente e úmido, com chuvas no verão e estiagem no inverno, segundo a classificação de Köppen (1948). As médias anuais de temperatura e precipitação pluviométrica foram $27{ }^{\circ} \mathrm{C}$ e $1.405 \mathrm{~mm}$, respectivamente. Existem duas estações climáticas bem definidas: uma seca (junho a agosto) com precipitação média de $55 \mathrm{~mm}$ e com temperatura média de $23^{\circ} \mathrm{C}$, outra chuvosa (novembro a março) com precipitação média de $1.066 \mathrm{~mm}$ e com temperatura média de $30^{\circ} \mathrm{C}$ (Diretoria Agrícola de Paulo de Faria, que dista cerca de $30 \mathrm{Km}$ da área de estudo).

Durante os meses secos algumas árvores perdem as folhas, o que classifica esta floresta como estacional semidecidual (Veloso 1991). O solo pertence à unidade taxonômica Latossolo Vermelho (LV), conhecido como terra-roxa (Embrapa 1999).

Com base em características fisionômicas, Stranghetti \& Ranga (1998) reconheceram três fitofisionomias na área de estudo. $\mathrm{Na}$ área onde o estudo foi realizado o ambiente é sombreado e aparentemente mais úmido, sendo considerada pelas autoras como a mais preservada da Estação Ecológica. A área selecionada tem cerca de 100 ha, com contorno irregular e maior dimensão no sentido sudeste-noroeste (figura 1). Coleta de dados - A coleta de dados para a descrição da comunidade de lianas foi realizada durante o período de março de 2000 a setembro de 2003, utilizando-se o método de parcelas (Mueller-Dombois \& Ellemberg 1974). Em uma área selecionada de quatro hectares foram delimitadas 100 parcelas de $10 \times 10 \mathrm{~m}$, totalizando uma área amostral de $10.000 \mathrm{~m}^{2}$ (1 ha).

Para alocar as parcelas foi traçado, em um mapa, um sistema de coordenadas com eixos ortogonais x e y de $200 \mathrm{~m}$ de comprimento. $\mathrm{O}$ eixo $\mathrm{x}$ correspondente a uma trilha que corta a área de estudo. Em seguida, foram sorteados pares Xy de números, os quais definiram a localização do vértice das parcelas voltado para a origem dos eixos. As parcelas foram posicionadas em campo por meio de bússola e trena, tomando a trilha como referência.

Quando as parcelas sorteadas se localizavam em áreas com árvores mortas (caídas ou em pé), sua alocação em campo era deslocada para a parcela mais próxima onde a amostragem era possível, seguindo a orientação do eixo y sentido norte. Essas áreas não foram consideradas devido a dificuldade de amostragem, apresentando densos emaranhados que impossibilitavam a individualização dos espécimes.

Em cada parcela foram numerados, medidos e identificados todos os indivíduos de lianas enraizados na parcela com DAP $\geq 1 \mathrm{~cm}$ e todos os indivíduos arbóreos com diâmetro a altura do peito (DAP) $\geq 3 \mathrm{~cm}$. As espécies de lianas e árvores coletadas foram identificadas com o auxílio de bibliografia especializada, comparadas com materiais depositados nos herbários SJRP, UEC e USP e, quando necessário, enviadas a especialistas. O material foi incorporado ao herbário SJRP do Departamento de Zoologia e Botânica do Instituto de Biociências Letras e Ciências Exatas, UNESP, São José do Rio Preto e UEC do Departamento de Botânica da UNICAMP.

Foi adotado o sistema de classificação APG II (2003) para a listagem de famílias, com exceção de Leguminosae que foi tratada de acordo com Judd et al. (2002). Os nomes de autores das espécies foram citados seguindo Brumitt \& Powells (1992). Análise de dados - Descrição da comunidade de lianas - Foram utilizados descritores absolutos e relativos de densidade, frequiência e dominância, e o índice de valor de importância (IVI) segundo Mueller-Dombois \& Ellenberg (1974). Curvas de acumulação de espécies/área amostrada e de incremento 

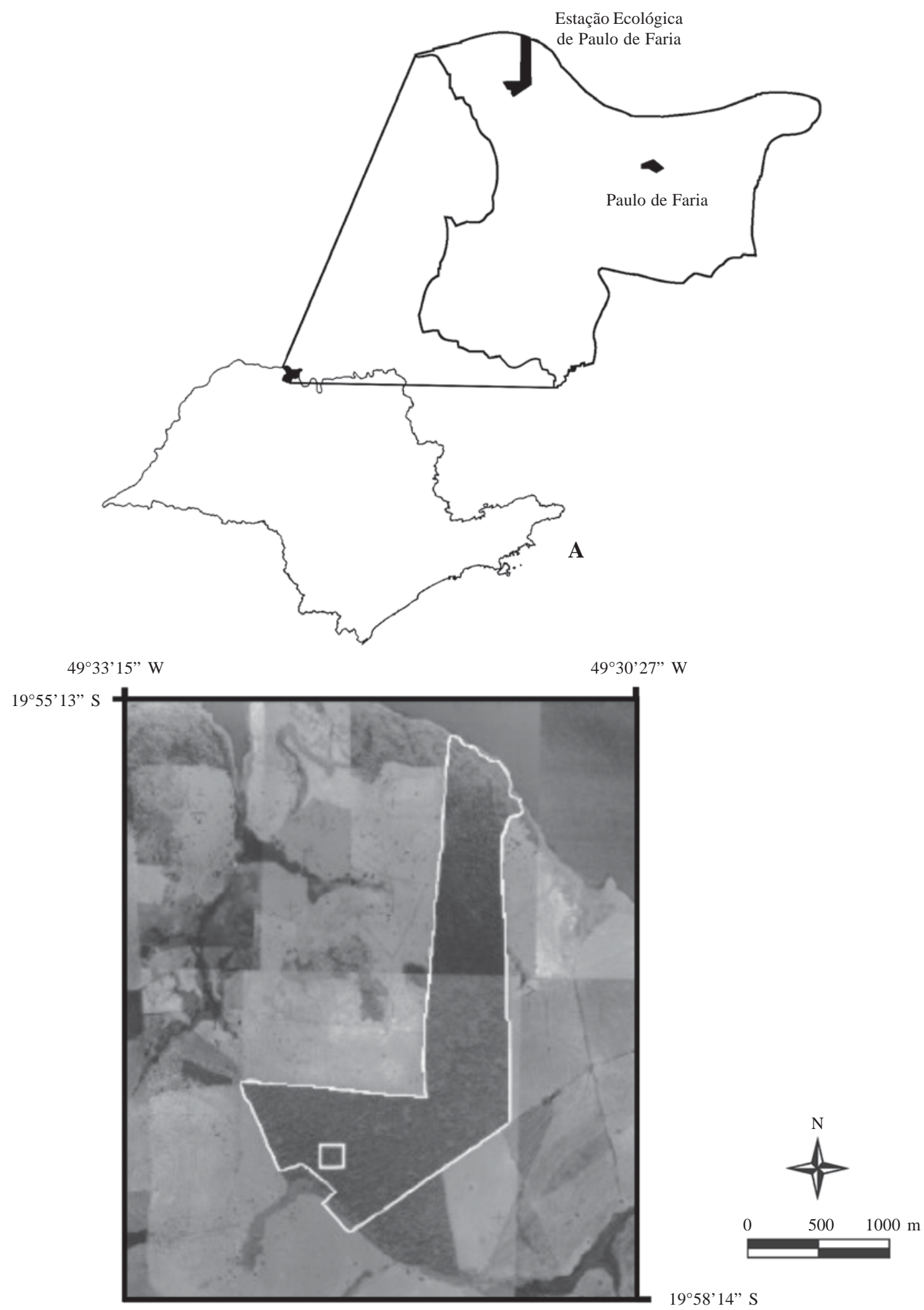

B

Figura 1. A. Localização geográfica do Município de Paulo de Faria, região norte do Estado de São Paulo - Brasil e localização da Estação Ecológica de Paulo de Faria (EEPF). B. Fotografia aérea do fragmento estudado com a localização do trecho amostrado (quadrado branco).

Figure 1. A. Geographic location of Paulo de Faria, in northern region of the state of São Paulo - Brazil and location of "Estação Ecológica de Paulo de Faria" (EEPF). B. Aerial photograph of the studied fragment, white square indicates the sampled area. 
de novas espécies/área foram construídas para árvores e lianas. Os índices de diversidade de Shannon-Weaver e o de equabilidade de Pielou (Pielou 1975) foram estimados para lianas e árvores. Para a construção das curvas e os cálculos dos índices, foi utilizado o programa EstimateS V.5 (Colwell 1997).

Para a verificação do padrão de distribuição espacial dos indivíduos de lianas utilizou-se o teste de Mantel (Pc-Ord V.3.14-McCune \& Mefford 1997). Nesta análise, correlacionouse a distância espacial em metros e a dissimilaridade em termos de abundância de indivíduos de lianas entre todos os pares de parcelas (utilizou-se distância Euclidiana para ambas variáveis). Distribuições agregadas seriam detectadas por correlações positivas, significando que parcelas mais próximas espacialmente apresentam abundâncias semelhantes de espécies de lianas.

Para verificar qual dos descritores (densidade, freqüência ou dominância) mais influenciou o índice de valor de importância das espécies de lianas (IVI), foi calculada a correlação de Pearson entre o IVI e os descritores.

\section{Resultados}

Riqueza de lianas - Ao todo foram amostrados 1427 indivíduos de 45 espécies de lianas, pertencentes a 13 famílias (tabela 1), sendo que as famílias mais ricas foram Bignoniaceae (14 espécies), Sapindaceae (nove espécies), Malpighiaceae (seis espécies) e Leguminosae (quatro espécies), o que corresponde a 73\% das espécies amostradas.

A tabela 2 resume as principais características estruturais das comunidades de lianas e de árvores (Rezende 2005) amostradas. A composição de espécies de lianas (45 espécies) foi aproximadamente igual à metade da composição de espécies de árvores (87 espécies). A curva de coletor e de taxa de incremento para lianas (figura 2) mostraram uma tendência à estabilização em aproximadamente $50 \%$ da área amostrada (0,5 ha). A curva de coletor para árvores não demonstrou de forma clara esta tendência de estabilização, porém a taxa de incremento de novas espécies foi menor a partir de 50 parcelas amostradas (figura 2).

Abundância de lianas - As famílias representadas pelos maiores números de indivíduos foram Bignoniaceae, Sapindaceae e Apocynaceae, reunindo 80\% do total.

As 10 espécies mais abundantes de lianas representaram $75 \%$ do total de indivíduos (tabela 3). Dentre estas Melloa quadrivalvis (Bignoniaceae) foi a espécie mais comum na área estudada, representando cerca de $13 \%$ do total de indivíduos de lianas amostrados, seguida de Forsteronia pilosa (12\%), F. tomentosa (11\%) (Apocynaceae) e Urvillea laevis (10\%) que juntas representam quase metade $(46 \%)$ do total de indivíduos amostrados.

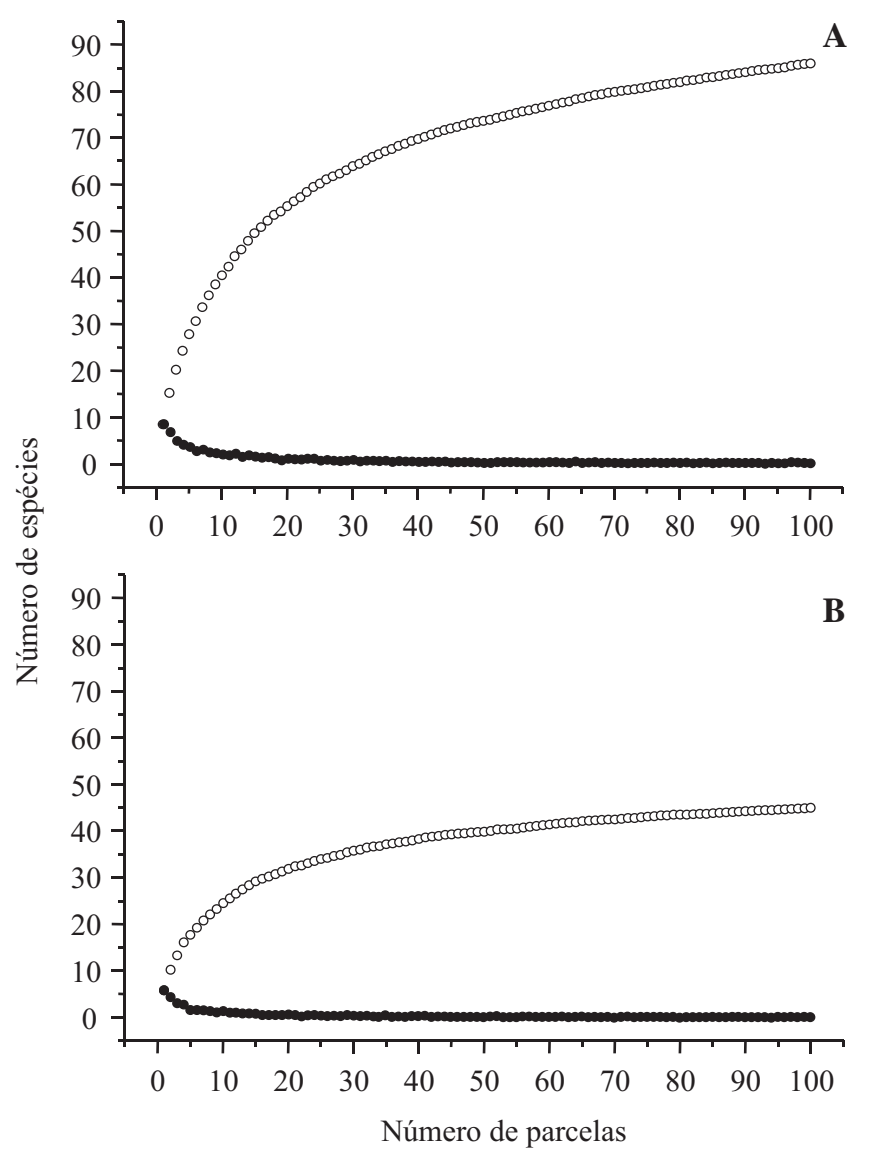

Figura 2. Curva do coletor e taxa de incremento de espécies de árvores (A) e lianas (B) em 1 ha de floresta estacional semidecidual na Estação Ecológica de Paulo de Faria, São Paulo, Brasil. ( $\circ=$ Riqueza observada; $\bullet=$ Incremento).

Figure 2. Collector's curve and rate of tree (A) and liana (B) species increment in 1 ha of seasonal semideciduous forest in the "Estação Ecológica de Paulo de Faria", São Paulo, Brazil. ( $\mathrm{o}=$ Observed richness; $\bullet=$ Increment $)$.

Distribuição de tamanho das lianas - Cerca de 58\% dos indivíduos amostrados apresentaram diâmetro <2,5 $\mathrm{cm}$, sendo assim, considerando a ocorrência das famílias de lianas em duas classes de diâmetro $(<2,5 \mathrm{~cm}$ e $\geq 2,5$ $\mathrm{cm})$, verificou-se que o padrão de riqueza não variou quanto aos diferentes diâmetros, sendo as famílias Bignoniaceae e Sapindaceae as mais ricas em ambas (tabela 4). Bignoniaceae e Sapindaceae foram também as mais abundantes, com destaque para a última, que apresentou maior abundância na classe de indivíduos com diâmetros menores (figura 3), em função do grande número de indivíduos de Urvillea laevis (tabela 5).

A distribuição em classes diamétricas dos indivíduos de lianas $\geq 1 \mathrm{~cm}$ apresentou-se na forma de $\mathrm{J}$ invertido representando mais da metade dos indivíduos $(57,6 \%)$ 
Tabela 1. Espécies de lianas amostradas em 1 ha de floresta estacional semidecidual na Estação Ecológica de Paulo de Faria, São Paulo, Brasil. Número do coletor de Andréia Alves Rezende, depositado no herbário SJRP.

Table 1. Lianas species sampled in 1 ha of a seasonal semideciduous forest in the "Estação Ecológica de Paulo de Faria", São Paulo, Brazil. Collector's number of Andréia Alves Rezende, deposited in the SJRP herbarium.

\begin{tabular}{|c|c|c|c|}
\hline Família & Espécie & $\begin{array}{l}\text { Mecanismo } \\
\text { de escalada }\end{array}$ & $\begin{array}{l}\text { Número do } \\
\text { coletor }\end{array}$ \\
\hline Amaranthaceae & Hebanthe paniculata Mart. & Volúvel & 742 \\
\hline \multirow[t]{4}{*}{ Apocynaceae } & Forsteronia pilosa (Vell.) Müll. Arg. & Volúvel & 667 \\
\hline & Forsteronia pubescens A. DC. & Volúvel & 720 \\
\hline & Schubertia sp. & Volúvel & 612 \\
\hline & Secondatia sp. & Volúvel & 780 \\
\hline Asteraceae & Dasyphyllum flagellare (Casar.) Cabrera & Escandente & 791 \\
\hline \multirow[t]{14}{*}{ Bignoniaceae } & Adenocalymma bracteatum (Cham.) DC. & Gavinha & 744 \\
\hline & Amphilophium paniculatum (L.) Kunth & Gavinha & 614 \\
\hline & Arrabidaea chica (Humb \& Bonpl.) Verl. & Gavinha & 683 \\
\hline & Arrabidaea leucopogon (Cham.) Sandwith & Gavinha & 741 \\
\hline & Arrabidaea pulchella (Cham.) Bureau & Gavinha & 664 \\
\hline & Arrabidaea selloi (Spreng.) Sandwith & Gavinha & 790 \\
\hline & Arrabidaea triplinervia (Mart. ex DC.) Baill. ex Bureau & Gavinha & 610 \\
\hline & Lundia obliqua Sond. & Gavinha & 768 \\
\hline & Macfadyena unguis-cati (L.) A.H. Gentry & Gavinha & 715 \\
\hline & Melloa quadrivalvis (Jacq.) A.H. Gentry & Gavinha & 711 \\
\hline & Paragonia pyramidata (Rich.) Bureau & Gavinha & 722 \\
\hline & Pleonotoma tetraquetrum (Cham.) Bureau & Gavinha & 792 \\
\hline & Pyrostegia venusta (Ker-Gawl.) Miers & Gavinha & 716 \\
\hline & Tynnanthus elegans Miers & Gavinha & 774 \\
\hline Combretaceae & Combretum discolor Taub. & Volúvel & 717 \\
\hline Cucurbitaceae & Wilbrandia longisepala Cogn. & Gavinha & 769 \\
\hline Celastraceae & Anthodon decussatum Ruiz \& Pav. & Gavinha & 721 \\
\hline \multirow[t]{4}{*}{ Leguminosae } & Bauhinia microstachya (Raddi) J. F. Macbr. & Gavinha & 710 \\
\hline & Machaerium oblongifolium Vogel $^{*}$ & Escandente & 725 \\
\hline & Rhynchosia phaseoloides (Sw.) DC. & Volúvel & 793 \\
\hline & Indeterminada & Volúvel & 767 \\
\hline \multirow[t]{6}{*}{ Malpighiaceae } & Banisteriopsis lutea (Griseb.) Cuatrec. & Volúvel & 771 \\
\hline & Heteropterys argyrophaea A. Juss. & Volúvel & 781 \\
\hline & Heteropterys dumetorum (Griseb.) Nied. & Volúvel & 743 \\
\hline & Hiraea sp. & Volúvel & 718 \\
\hline & Mascagnia chlorocarpa (A. Juss.) Griseb. & Volúvel & 745 \\
\hline & Tetrapterys sp. & Volúvel & 766 \\
\hline Menispermaceae & Cissampelos pareira $\mathrm{L}$. & Volúvel & 779 \\
\hline Rubiaceae & Guettarda guianensis Urb.* & Escandente & 762 \\
\hline \multirow[t]{9}{*}{ Sapindaceae } & Cardiospermum grandiflorum Sw. & Gavinha & 723 \\
\hline & Serjania caracasana (Jacq.) Willd. & Gavinha & 620 \\
\hline & Serjania communis Cambess. & Gavinha & 803 \\
\hline & Serjania hebecarpa Benth. & Gavinha & 688 \\
\hline & Serjania laruotteana Cambess. & Gavinha & 654 \\
\hline & Serjania lethalis A. St.-Hil. & Gavinha & 656 \\
\hline & Serjania sp. & Gavinha & 782 \\
\hline & Urvillea laevis Radlk. & Gavinha & 661 \\
\hline & Urvillea uniloba Radlk. & Gavinha & 739 \\
\hline Vitaceae & Cissus campestris (Baker) Planch. & Gavinha & 670 \\
\hline
\end{tabular}

* Hábito de liana e também como árvore ou arbusto.

* Habit liana and tree or shrub. 
Tabela 2. Descritores estruturais da comunidade de lianas $\geq 1$ $\mathrm{cm}$ (DAP) e de árvores $\geq 3 \mathrm{~cm}$ (DAP) em 1 ha de floresta estacional semidecidual na Estação Ecológica de Paulo de Faria, São Paulo, Brasil.

Table 2. Liana and tree community parameters: lianas $\geq 1 \mathrm{~cm}$ $(\mathrm{DBH})$ and tree $\geq 3 \mathrm{~cm}(\mathrm{DBH})$ in 1 ha of seasonal semideciduous forest in the "Estação Ecológica de Paulo de Faria", São Paulo, Brazil.

\begin{tabular}{lcc}
\hline Variáveis & Lianas & Árvores \\
\hline Número de espécies & 45 & 87 \\
Número de gêneros & 33 & 68 \\
Número de famílias & 13 & 35 \\
Equabilidade (Pielou) & 0,78 & 0,80 \\
Índice de diversidade (nats & 2,98 & 3,58 \\
indivíduo-1) (Shannon-Weaver) & & \\
Densidade (ind ha-1) & 1427 & 1419 \\
Área basal $\left(\mathrm{m}^{2} \mathrm{ha}^{-1}\right)$ & 1,73 & 30,27 \\
\hline
\end{tabular}

cujos diâmetros estão entre 1-2,5 cm (figura 4). Algumas espécies (13\%) apresentam somente indivíduos com diâmetro menor que $2,5 \mathrm{~cm}$.

Somente 12 espécies e 26 indivíduos apresentaram o diâmetro $>10 \mathrm{~cm}$, dos quais sete espécies e 19 indivíduos pertencem à família Bignoniaceae. $\mathrm{O}$ maior indivíduo pertenceu a Melloa quadrivalvis (Bignoniaceae), com diâmetro de $30,6 \mathrm{~cm}$.

Parâmetros estruturais - Em geral, as espécies com maior densidade são as mais frequientes (tabela 3). Nenhuma espécie de liana ocorreu em todas as parcelas e somente cinco (Forsteronia pubescens, Urvillea laevis, Melloa quadrivalvis, Arrabidaea triplinervia e Forsteronia pilosa) foram encontradas em mais de $40 \%$ das parcelas, sendo estas cinco espécies as mais abundantes. As demais espécies ocorreram em freqüências menores, porém, considerando a análise de distribuição espacial, apenas Arrabidaea chica $(r=0,07 ; P=0,04 ; n=56$ indivíduos) e Combretum discolor $(r=0,13 ; P=0,001$; $n=38$ indivíduos) apresentaram um padrão agregado.

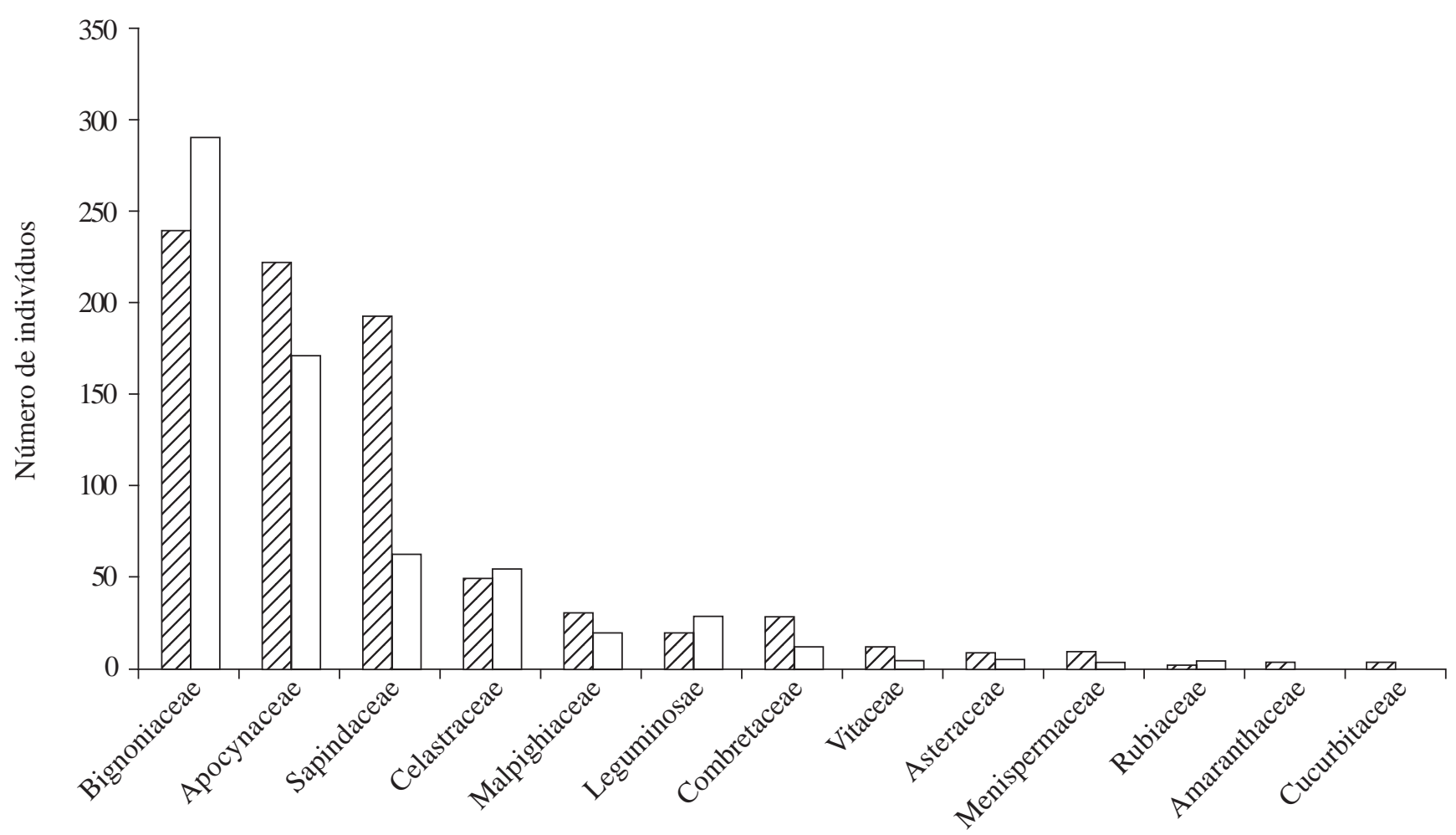

Figura 3. Número de indivíduos de lianas por famílias em duas classes de tamanho em 1 ha de floresta estacional semidecidual na Estação Ecológica de Paulo de Faria, São Paulo, Brasil $(Z / \lambda=\mathrm{DAP}<2,5 \mathrm{~cm} ; \square=\mathrm{DAP}>2,5 \mathrm{~cm})$.

Figure 3. Number of liana individuals per families in two classes of size in 1 ha of seasonal semideciduous forest in the "Estação Ecológica de Paulo de Faria", São Paulo, Brazil ( $\angle \lambda=\mathrm{DAP}<2,5 \mathrm{~cm} ; \square=\mathrm{DAP}>2,5 \mathrm{~cm})$. 
As 10 espécies de lianas que apresentaram maior dominância representam cerca de $80 \%$ da área basal total (tabela 3). Em geral as espécies com maior dominância foram também as com maior densidade.

A tabela 3 mostra os índices de valor de importância para as espécies de lianas (IVI) amostradas. Nas 20 espécies mais importantes, o índice de valor de importância foi mais influenciado pela abundância $\left(r_{\text {Pearson }}=0,94\right)$ do que pela freqüência $\left(r_{\text {Pearson }}=0,83\right)$ e área basal $\left(r_{\text {Pearson }}=0,80\right)$.

\section{Discussão}

No fragmento estudado, as famílias Bignoniaceae, Sapindaceae, Malpighiaceae e Leguminosae foram as mais ricas em espécies de lianas, semelhante a outras florestas neotropicais (Gentry 1991), sendo que poucas famílias agrupam muitas espécies. Entretanto, a proporção de espécies pertencentes a estas quatro famílias (73\%) neste trabalho, foi maior do que a já verificada nos neotrópicos, entre $64 \%$ e $69 \%$ para as 10 famílias mais ricas, com a ressalva de que os critérios de amostragem foram diferentes (Gentry 1991).
O índice de diversidade de lianas nem sempre é mencionado nos estudos em florestas tropicais, o que dificulta o estabelecimento de comparações entre estes resultados, conforme observado por Putz (1984) e Gentry (1991). No presente estudo, o índice de diversidade de Shannon-Weaver $\left(H^{\prime}=2,98\right)$ foi significativamente inferior, de acordo com o teste $t$ de Hutcheson $\left(t_{943}=\right.$ -4,121; $P<10^{-4}-$ Zar 1996), ao de outra floresta semidecídua do Estado de São Paulo $\left(H^{\prime}=3,20\right.$ - Hora $\&$ Soares 2002). Embora o valor de equabilidade em nosso trabalho tenha sido relativamente alto $(\mathrm{J}=0,78)$, a densidade das espécies foi desigual, com quatro muito abundantes: Melloa quadrivalvis, Forsteronia pilosa, F. pubescens e Urvillea laevis (46\% dos indivíduos amostrados). No entanto, a falta de informações sobre as características ecológicas destas espécies não permite discutir sua elevada abundância na área de estudo.

Os resultados apontaram que a área estudada apresentou densidade e riqueza de espécies de lianas (50\% dos indivíduos e 34\% das espécies amostradas) superiores ao valor médio de aproximadamente $25 \%$ em relação às outras espécies lenhosas, normalmente relatadas em florestas tropicais (Gentry 1991, Appanah

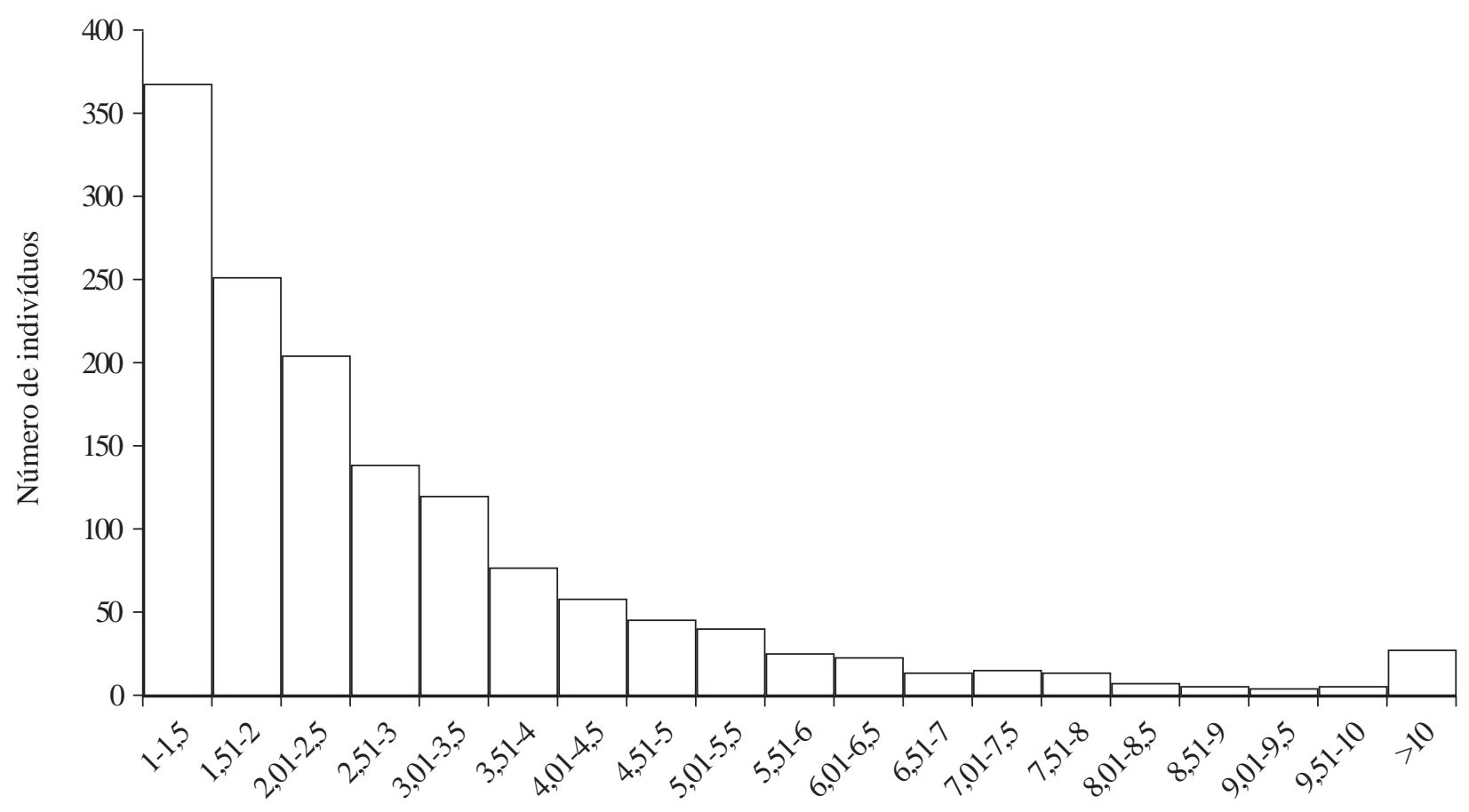

Figura 4. Distribuição do número de indivíduos de lianas em classes de diâmetro em 1 ha de floresta estacional semidecidual na Estação Ecológica de Paulo de Faria, São Paulo, Brasil.

Figure 4. Distribution of the number of liana individuals per diameter classes in 1 ha of seasonal semideciduous forest in the "Estação Ecológica de Paulo de Faria", São Paulo, Brazil. 
Tabela 3. Descritores fitossociológicos das espécies de lianas amostradas em 1 ha de floresta estacional semidecidual da Estação Ecológica de Paulo de Faria, São Paulo, Brasil. DA = densidade absoluta; FA = frequiência absoluta; DoA = dominância absoluta; $\mathrm{DR}$ = densidade relativa; $\mathrm{FR}$ = freqüência relativa; $\mathrm{DoR}$ = dominância relativa; IVI = índice de valor de importância.

Table 3. Phytosociological parameters of the lianas species sampled in 1 ha of seasonal semideciduous forest in the "Estação Ecológica de Paulo de Faria", São Paulo, Brazil. DA = absolute density; FA = absolute frequency; DoA = absolute dominance; $\mathrm{DR}=$ relative density $; \mathrm{FR}=$ relative frequency $; \mathrm{DoR}=$ relative dominance $\mathrm{IVI}=$ index of value of importance .

\begin{tabular}{|c|c|c|c|c|c|c|c|c|}
\hline Espécie & DA & $\begin{array}{c}\text { FA } \\
\left(\text { ind ha }^{-1} \text { ) }\right.\end{array}$ & $\begin{array}{c}\text { DoA } \\
\left(\mathrm{m}^{2} \mathrm{ha}^{-1}\right)\end{array}$ & $\begin{array}{c}\mathrm{DR} \\
\%\end{array}$ & $\begin{array}{c}\text { FR } \\
\%\end{array}$ & $\begin{array}{c}\text { DoR } \\
\%\end{array}$ & $\begin{array}{l}\text { Diâmetro } \\
\text { máximo }(\mathrm{cm})\end{array}$ & IVI \\
\hline Melloa quadrivalvis & 188 & 48 & 0,3433 & 13,17 & 8,19 & 19,84 & 30,60 & 41,21 \\
\hline Arrabidaea triplinervia & 121 & 44 & 0,2666 & 8,48 & 7,51 & 15,41 & 30,00 & 31,40 \\
\hline Forsteronia pilosa & 168 & 66 & 0,0979 & 11,77 & 11,26 & 5,66 & 14,00 & 28,70 \\
\hline Forsteronia pubescens & 171 & 40 & 0,1611 & 11,98 & 6,83 & 9,31 & 13,00 & 28,12 \\
\hline Urvillea laevis & 143 & 61 & 0,0402 & 10,02 & 10,41 & 2,33 & 7,72 & 22,76 \\
\hline Anthodon decussatum & 101 & 35 & 0,1045 & 7,08 & 5,97 & 6,04 & 10,20 & 19,09 \\
\hline Bauhinia microstachya & 40 & 30 & 0,0874 & 2,80 & 5,12 & 5,05 & 18,91 & 12,97 \\
\hline Arrabidaea chica & 56 & 20 & 0,0884 & 3,92 & 3,41 & 5,11 & 12,86 & 12,45 \\
\hline Serjania hebecarpa & 18 & 12 & 0,1094 & 1,26 & 2,05 & 6,33 & 23,91 & 9,64 \\
\hline Adenocalymna bracteatum & 28 & 11 & 0,0840 & 1,96 & 1,88 & 4,85 & 21,10 & 8,69 \\
\hline Serjania lethalis & 35 & 23 & 0,0369 & 2,45 & 3,92 & 2,13 & 7,74 & 8,51 \\
\hline Combretum discolor & 38 & 23 & 0,0171 & 2,66 & 3,92 & 0,99 & 6,10 & 7,58 \\
\hline Secondatia sp. & 45 & 12 & 0,0195 & 3,15 & 2,05 & 1,13 & 6,30 & 6,33 \\
\hline Pyrostegia venusta & 11 & 9 & 0,0632 & 0,77 & 1,54 & 3,65 & 25,79 & 5,96 \\
\hline Amphilophium paniculatum & 24 & 13 & 0,0251 & 1,68 & 2,22 & 1,45 & 7,50 & 5,35 \\
\hline Arrabidaea selloi & 15 & 9 & 0,0403 & 1,05 & 1,54 & 2,33 & 9,70 & 4,91 \\
\hline Macfadyena unguis-cati & 15 & 12 & 0,0229 & 1,05 & 2,05 & 1,33 & 12,67 & 4,43 \\
\hline Arrabidaea leисороgon & 20 & 9 & 0,0180 & 1,40 & 1,54 & 1,04 & 5,44 & 3,98 \\
\hline Urvillea uniloba & 19 & 5 & 0,0282 & 1,33 & 0,85 & 1,63 & 9,20 & 3,81 \\
\hline Mascagnia chlorocarpa & 18 & 11 & 0,0071 & 1,26 & 1,88 & 0,41 & 4,20 & 3,55 \\
\hline Cissus campestris & 14 & 11 & 0,0054 & 0,98 & 1,88 & 0,31 & 4,38 & 3,17 \\
\hline Serjania caracasana & 12 & 10 & 0,0058 & 0,84 & 1,71 & 0,33 & 5,50 & 2,88 \\
\hline Banisteriopsis lutea & 12 & 7 & 0,0138 & 0,84 & 1,19 & 0,80 & 8,30 & 2,83 \\
\hline Lundia obliqua & 17 & 5 & 0,0089 & 1,19 & 0,85 & 0,52 & 5,40 & 2,56 \\
\hline Hiraea sp. & 12 & 9 & 0,0003 & 0,84 & 1,54 & 0,02 & 4,64 & 2,39 \\
\hline Tynnanthus elegans & 12 & 5 & 0,0053 & 0,84 & 0,85 & 0,30 & 4,30 & 2,00 \\
\hline Cissampelos pareira & 10 & 6 & 0,0045 & 0,70 & 1,02 & 0,26 & 5,30 & 1,98 \\
\hline Dasyphyllum flagellare & 9 & 6 & 0,0047 & 0,63 & 1,02 & 0,27 & 4,00 & 1,93 \\
\hline Serjania laruotteana & 9 & 5 & 0,0031 & 0,63 & 0,85 & 0,18 & 3,40 & 1,66 \\
\hline Pleonotoma tetraquetrum & 4 & 4 & 0,0017 & 0,28 & 0,68 & 0,10 & 3,05 & 1,06 \\
\hline Cardiospermum grandiflorum & 9 & 2 & 0,0010 & 0,63 & 0,34 & 0,06 & 1,45 & 1,03 \\
\hline Paragonia pyramidata & 6 & 2 & 0,0020 & 0,42 & 0,34 & 0,12 & 3,48 & 0,88 \\
\hline Arrabidaea pulchella & 4 & 3 & 0,0013 & 0,28 & 0,51 & 0,07 & 2,94 & 0,87 \\
\hline Serjania communis & 3 & 3 & 0,0009 & 0,21 & 0,51 & 0,05 & 2,40 & 0,77 \\
\hline Heteropterys dumetorum & 1 & 1 & 0,0080 & 0,07 & 0,17 & 0,46 & 1,22 & 0,71 \\
\hline Guettarda guianensis & 2 & 2 & 0,0022 & 0,14 & 0,34 & 0,13 & 3,40 & 0,61 \\
\hline Serjania sp. & 2 & 2 & 0,0009 & 0,14 & 0,34 & 0,05 & 2,30 & 0,53 \\
\hline Rhynchosia phaseoloides & 2 & 2 & 0,0008 & 0,14 & 0,34 & 0,04 & 2,50 & 0,53 \\
\hline Tetrapterys sp. & 2 & 2 & 0,0007 & 0,14 & 0,34 & 0,04 & 2,40 & 0,52 \\
\hline Heteropterys argyrophaea & 4 & 1 & 0,0001 & 0,28 & 0,17 & 0,01 & 3,10 & 0,46 \\
\hline Machaerium oblongifolium & 1 & 1 & 0,0021 & 0,07 & 0,17 & 0,12 & 5,15 & 0,36 \\
\hline Hebante paniculata & 2 & 1 & 0,0005 & 0,14 & 0,17 & 0,03 & 1,74 & 0,34 \\
\hline Wilbrandia longisepala & 2 & 1 & 0,0003 & 0,14 & 0,17 & 0,01 & 1,30 & 0,33 \\
\hline Leguminosae & 1 & 1 & 0,0011 & 0,07 & 0,17 & 0,06 & 1,91 & 0,30 \\
\hline Schubertia sp. & 1 & 1 & 0,0006 & 0,07 & 0,17 & 0,03 & 2,70 & 0,27 \\
\hline Total & 1427 & 586 & 1,7370 & 100,00 & 100,00 & 100,00 & - & 300,00 \\
\hline
\end{tabular}


et al. 1993). A abundância e a riqueza de lianas observadas foram semelhantes à de outra floresta estacional semidecidual no Estado de São Paulo estudada por Hora \& Soares (2002). Porém, considerando-se os valores extremos para as florestas tropicais, os dados sugerem que a comunidade de lianas da EEPF apresenta valores intermediários para estes parâmetros (riqueza e abundância), sendo que em florestas mais pobres as lianas podem representar 10\% das espécies lenhosas (Appanah et al. 1993), ao passo que, em florestas mais ricas, este valor pode ser de até 44\% (Pérez-Salicrup 2001). A razão da riqueza de liana/árvore $(0,51)$ foi superior aos valores que variam entre 0,10 a 0,40 , relatados na literatura (Putz 1984, Gentry 1988, Appanah et al. 1993, Wright et al. 1997). Assim, os resultados sugerem que a EEPF é relativamente rica em lianas. Deve ser considerado, ainda, que estes parâmetros (riqueza e densidade) possam ter sido subestimados, uma vez que as clareiras, mais ricas em lianas (Putz 1984), não foram amostradas. Ressaltamos, no entanto, que os valores observados de densidade de lianas devem ser interpretados com cautela, visto que a abundância de indivíduos nas amostras é influenciada pelo critério de inclusão, o que pode variar entre os estudos, levando a resultados muito diferentes dificultando comparações entre eles.

Considerando-se as classes diamétricas os indivíduos com DAP $\geq 2,5 \mathrm{~cm}$ estimou uma riqueza $18 \%$ menor que a observada incluindo todos os indivíduos. Por outro lado, considerando-se apenas os indivíduos com DAP $<2,5 \mathrm{~cm}$, estimou-se $95 \%$ da riqueza total da amostra. Esta variação também foi observada em Yasuní-Equador (Nabe-Nielsen 1998, Nabe-Nielsen 2001), porém, de forma mais acentuada, com o número médio de espécies por transecto de 0,1 ha diminuindo de 34,8 para 13 espécies, se fossem excluídos os indivíduos $<2,5 \mathrm{~cm}$. Na Índia, Parthasarathy et al. (2004) verificou que quando na amostragem diminuiu-se o diâmetro de $2 \mathrm{~cm}$ para $1 \mathrm{~cm}$ ocorreu um aumento na riqueza de $12 \%$ para $29 \%$ e na densidade de $22 \%$ para $71 \%$. Esta alta porcentagem de espécies excluídas deve-se às características morfológicas das lianas, que, em geral, apresentam diâmetros menores que outras espécies vegetais de hábito ereto.

Para o estudo de lianas o critério de inclusão $\geq 2,5$ $\mathrm{cm}$ parece não ser muito adequado para se estimar a riqueza, uma vez que é restritivo, o que é corroborado por Kurzel et al. (2006) que sugeriram um diâmetro mínimo de $2 \mathrm{~cm}$ para estimar a abundância e a diversidade de lianas. Assim, se o objetivo for estimar apenas a riqueza, a amostragem de indivíduos com DAPs entre 1 e $2 \mathrm{~cm}$ já seria suficiente, uma vez que se estimaria grande parte da riqueza com um pouco mais
Tabela 4. Número de espécies de lianas por famílias segundo a classe de diâmetro, em 1 ha de floresta estacional semidecidual na Estação Ecológica de Paulo de Faria, São Paulo, Brasil.

Table 4. Number of liana species per families according to diameter class, in 1 ha of seasonal semideciduous forest in the "Estação Ecológica de Paulo de Faria", São Paulo, Brazil.

\begin{tabular}{lccc}
\hline Família & $\begin{array}{c}\text { Total de } \\
\text { espécies }\end{array}$ & $\begin{array}{c}\text { DAP } \\
<2,5 \mathrm{~cm}\end{array}$ & $\begin{array}{c}\text { DAP } \\
\geq 2,5 \mathrm{~cm}\end{array}$ \\
\hline Bignoniaceae & 14 & 14 & 14 \\
Sapindaceae & 9 & 9 & 7 \\
Malpighiaceae & 6 & 6 & 3 \\
Leguminosae & 4 & 3 & 4 \\
Apocynaceae & 4 & 3 & 4 \\
Asteraceae & 1 & 1 & 1 \\
Combretaceae & 1 & 1 & 1 \\
Celastraceae & 1 & 1 & 1 \\
Menispermaceae & 1 & 1 & 1 \\
Rubiaceae & 1 & 1 & 1 \\
Vitaceae & 1 & 1 & 1 \\
Amaranthaceae & 1 & 1 & 0 \\
Cucurbitaceae & 1 & 1 & 0 \\
\hline Total & 45 & 43 & 38 \\
\hline
\end{tabular}

Tabela 5. Porcentagem de indivíduos com DAP $>1 \mathrm{e}<2,5 \mathrm{~cm}$ em relação ao número total de indivíduos nas 10 espécies mais abundantes de lianas em 1 ha de floresta estacional semidecidual na Estação Ecológica de Paulo de Faria, São Paulo, Brasil.

Table 5. Percentage of individuals with DBH $>1$ and $<2,5 \mathrm{~cm}$ with respect to the total number of individuals among the 10 most abundant species of lianas in 1 ha of seasonal semideciduous forest in the "Estação Ecológica de Paulo de Faria", São Paulo, Brazil.

\begin{tabular}{lcrrr}
\hline \multirow{2}{*}{ Espécies } & $\begin{array}{c}\text { Número total de } \\
\text { indivíduos de } \\
\text { cada espécie }\end{array}$ & DAP $<2,5 \mathrm{~cm}$ \\
\hline Melloa quadrivalvis & 188 & 85 & 45,2 \\
Forsteronia pilosa & 171 & 109 & 63,7 \\
Forsteronia pubescens & 168 & 76 & 45,2 \\
Urvillea laevis & 143 & 132 & $\mathbf{9 2 , 3}$ \\
Arrabidaea triplinervia & 121 & 40 & 33,1 \\
Anthodon decussatum & 101 & 48 & 47,5 \\
Arrabidaea chica & 56 & 20 & 35,7 \\
Secondatia sp. & 45 & 35 & 77,8 \\
Bauhinia microstachya & 40 & 16 & 40,0 \\
Combretum discolor & 38 & 27 & 71,1 \\
\hline
\end{tabular}


da metade do esforço amostral utilizado na amostragem total (DAP > $1 \mathrm{~cm}$ ). Contudo, se o enfoque for estimar a abundância de indivíduos, nossos dados sugerem que todos os indivíduos com DAP $>1 \mathrm{~cm}$ devem ser amostrados. Nossas proposições sobre distribuição de tamanhos estão de acordo com o sugerido por NabeNielsen (1998), em que o diâmetro $>1 \mathrm{~cm}$ seria mais apropriado para o estudo de lianas. Deve-se ressaltar a importância da padronização dos critérios de amostragem para permitir comparações mais conclusivas entre os resultados de diferentes estudos, embora a utilização de critérios de inclusão menores represente aumento do esforço amostral e exija maior experiência taxonômica para a identificação das plantas mais jovens.

Dentre os parâmetros analisados, o IVI, a dominância e a frequiência foram influenciados principalmente pela densidade, fato este atribuído à grande densidade de indivíduos pequenos. A maior influência da densidade diferiu do observado em outros estudos, em que a área basal foi o parâmetro mais importante (Hora \& Soares 2002, Muthuramkumar \& Parthasarathy 2000). Os resultados de frequiência merecem destaque, pois apenas duas espécies (Arrabidaea chica e Combretum discolor) apresentaram distribuição agregada, padrão este que pode ser atribuído ao crescimento clonal, comum em lianas (Caballé 1993, Peñalosa 1984, Putz 1984). Entretanto, esses resultados devem ser interpretados com cautela, pois o padrão espacial pode variar de acordo com a escala analisada. Informações detalhadas da história de vida das espécies, da dispersão de sementes e da propagação vegetativa, combinadas à dinâmica de clareiras, ajudariam a explicar de forma mais conclusiva o padrão de agregação de lianas.

A comparação desta comunidade de lianas com outros trabalhos publicados é atualmente muito difícil em função da falta de padronização no delineamento amostral e do critério de inclusão empregado. Em muitos estudos, as parcelas são distribuídas de forma contígua, o que possibilita estudar a comunidade e estabelecer relações com o gradiente ambiental. Outros estudos utilizam alocações aleatórias das parcelas, possibilitando maximizar a riqueza de espécies. A falta de padrão no tamanho das parcelas, no critério de inclusão, nas estratégias de amostragem (inclusão ou não de indivíduo clonal) e na definição de lianas (considerar ou não hemiepifitas), somada à baixa disponibilidade de dados quantitativos sobre as lianas, muitas vezes, impossibilita as comparações, como já mencionado. Sendo assim, existe uma urgente necessidade de padronizar alguns critérios de investigação em lianas para facilitar comparações regionais e inter-regionais (Gerwing et al. 2006). Os resultados deste estudo corroboram esta perspectiva, ao evidenciar as diferenças observadas quando critérios de inclusão diferentes são analisados separadamente.

$\mathrm{O}$ uso do protocolo sugerido por Gerwing et al. (2006) facilitaria comparações de dados coletados independentemente e o entendimento dos padrões de abundância, diversidade, biomassa e dinâmica.

O conhecimento da diversidade e estrutura de lianas nas florestas mostra-se útil para o planejamento de estratégias de manejo, auxiliando na determinação dos efeitos de distúrbios e do estado de sucessão dos fragmentos florestais.

Agradecimentos - Agradecemos ao Instituto Florestal pela autorização concedida para realização deste trabalho, ao Marco Antonio de Assis (Bignoniaceae) pela identificação taxonômica, ao Aparecido Donizete Thiago da Maia pela ajuda nos trabalhos de campo. A primeira autora foi financiada pelo CNPq (bolsa de doutorado).

\section{Referências bibliográficas}

APG II. 2003. An update of the Angiosperm Phylogeny Group classification for the orders and families of flowering plants: APG II. Botanical Journal of the Linnean Society 141:399-436.

APPANAH, S., GENTRY, A.H. \& LAFRANKIE, J.V. 1993. Liana diversity and species richness of Malaysian rain forest. Journal Tropical Forest Science 6:116-123.

BALFOUR, D.A. \& BOND, W.J. 1993. Factors limiting climber distribution and abundance in a southern African forest. Journal Ecology 81:93-99.

BRUMMITT, R.K. \& POWELLS, C.E. 1992. Authors of plant names. Royal Botanic Gardens, Kew.

CABALLÉ, G. 1993. Liana structure, function and selection: a comparative study of xylem cylinders of tropical rain forest species in Africa and America. Botanical Journal of the Linnean Society 113:41-60

COLWELL, R.K. 1997. EstimateS: statistical estimation of species richness and shared species from samples. Version 5. User's guide and application published at: http://viceroy.eeb. uconn.edu/estimates (acesso em 22/09/2003).

EMBRAPA - CNPS. 1999. Sistema brasileiro de classificação de solos. Empresa Brasileira de Pesquisa Agropecuária, Centro Nacional de Pesquisa de Solos, Rio de Janeiro.

ENGEL, V.L., FONSECA, R.C.B. \& OLIVEIRA, R.E. 1998. Ecologia de lianas e o manejo de fragmentos florestais. Série Técnica IPEF 12:43-64.

GENTRY, A.H. 1988. Changes in plant community diversity and floristic composition on environmental and geographical gradients. Annals of the Missouri Botanical Garden 75:1-34.

GENTRY, A.H. 1991. The distribution and evolution of climbing plants. In The Biology of Vines (F. E. Putz \& H. A. Mooney, eds.). Cambridge University Press, Cambridge, p. 3-49. 
GERWING, J.J., SCHNITZER, S.A., BURNHAM, R.J., BONGERS, F., CHAVE, F., DEWALT, S.J., EWANGO, C.E.N., FOSTER, R., KENFACK, D., MARTÍNEZ-RAMOS, M., PARREN, M., PARTHASARATHY, N., PÉREZ-SALICRUP, D.R., PUTZ, F.E. \& THOMAS, D.W. 2006. A standard protocol for liana censuses. Biotropica 38:256-261.

HEGARTY, E.E. \& CABALLÉ, G. 1991. Distribution and abundance of vines in forest communities. In The Biology of Vines (F.E. Putz \& H.A. Mooney, eds.). Cambridge University Press, Cambridge, p. 313-335.

HORA, R.C. \& SOARES, J.J. 2002. Estrutura fitossociológica da comunidade de lianas em uma floresta estacional semidecidual na Fazenda Canchim, São Carlos, SP. Revista Brasileira de Botânica 25:323-329.

JOHNS, A.D. 1988. Effects of "selective" timber extraction on rain forest structure and composition and some consequences for frugivores and folivores. Biotropica 20:31-37

JUDD, W.S., CAMPBELL, C.S., KELLOGG, E.A. \& STEVENS. P.F. 2002. Plant systematics: A phylogenetic approach. Sinauer Associates, Inc., Sunderland.

KIM, A.C. 1996. Lianas da Mata Atlântica do Estado de São Paulo. Dissertação de mestrado, Universidade Estadual de Campinas, Campinas.

KÖPPEN, W. 1948. Climatologia. Fundo de Cultura Econômica. México.

KURZEL, B.P., SCHNITZER, S.A. \& CARSON, W.P. 2006. Predicting liana crown location from stem diameter in three Panamanian Lowland Forests. Biotropica 38: 262-266.

LOMBARDI, J.A., TEMPONI, L.G., LEITE, C.A. 1999. Mortality and diameter growth of lianas in a semideciduous forest fragment in Southeastern Brazil. Acta Botanica Brasilica 13:159-165.

MCCUNE, B. \& MEFFORD, M.J. 1997. Multivariate analysis of ecological data. Version 3.14 MjM Software, Gleneden Beach, Oregon.

MORELLATO, L.P. \& LEITÃO FILHO, H.F. 1996. Reproductive phenology of climbers in a Southeastern Brazilian Forest. Biotropica 28:180-191.

MORELLATO, L.P. \& LEITÃO FILHO, H.F. 1998. Levantamento florístico da comunidade de trepadeiras de uma floresta semidecídua no sudeste do Brasil. Boletim do Museu Nacional do Rio de Janeiro - série botânica 103: 1-15.

MUELLER-DOMBOIS, D. \& ELLEMBERG, H. 1974. Aims and methods of vegetation ecology. John Wiley, New York.

MUTHURAMKUMAR, S. \& PARTHASARATHY,N. 2000. Alpha diversity of lianas in a tropical evergreen forest in Anamalais, Western Ghats, India. Diversity and Distributions 6:1-14.

NABE-NIELSEN, J. 1998. Lianernes diversitet og plantefordelende faktorer i Yasuní, Ecuador. Del A - Thesis Progress Report. Aarhus University, Denmark.

NABE-NIELSEN, J. 2001. Diversity and distribution of lianas in a neotropical rain forest, Yasuní National Park, Ecuador. Journal of Tropical Ecology 17:1-19.
PARTHASARATHY, N., MUTHURAMKUMAR, S. \& REDDY, M.S. 2004. Patterns of liana diversity in tropical evergreen forests of peninsular India. Forest Ecology and Management 190:15-31.

PEÑALOSA, J. 1984. Basal branching and vegetative spread in two tropical rain forest lianas. Biotropica 16:1-9.

PÉREZ-SALICRUP, D.R. 2001. Effect of liana cutting on tree regeneration in a liana forest in Amazonian Bolivia. Ecology 82:389-396.

PÉREZ-SALICRUP, D.R., SCHNITZER, S.A. \& PUTZ, F.E. 2004. Community ecology and management of lianas. Forest Ecology and Management 190:1-2.

PIELOU, E.C. 1975. Ecological Diversity. Wiley and Sons, New York.

PUTZ, F.E. 1984. The natural history of lianas on Barro Colorado Island, Panama. Ecology 65:1713-1724.

REZENDE, A.A. 2005. Comunidade de lianas e sua associação com árvores em uma Floresta Estacional Semidecidual. Tese de doutorado, Universidade de Campinas, Campinas.

REZENDE, A.A. \& RANGA, N.T. 2005. Lianas da Estação Ecológica do Noroeste Paulista, São José do Rio Preto/ Mirassol, SP. Acta Botanica Brasilica. 19:273-279.

RICE, K., BROKAW, N. \& THOMPSON, J. 2004. Liana abundance in a Puerto Rican forest. Forest Ecology and Management 190:32-42.

ROZZA, A. 2003. Manejo e regeneração de trecho degradado de floresta estacional semidecidual: Reserva municipal de Santa Genebra, Campinas, SP. Tese de Doutorado. Universidade Estadual de Campinas, Campinas.

SCHNITZER, S.A. 2005. A mechanistic explanation for global patterns of liana abundance and distribution. The American Naturalist 166:262-276.

SCHNITZER, S.A. \& BONGERS, F.A. 2002. The ecology of lianas and their role in forests. Trends in Ecology \& Evolution 17:223-230.

STRANGHETTI, V. \& RANGA, N.T. 1998. Levantamento florístico das espécies vasculares de uma floresta estacional mesófila semidecídua da Estação Ecológica de Paulo de Faria, SP. Revista Brasileira de Botânica 21:289-298.

TONIATO, M.T.Z. \& OLIVEIRA-FILHO, A.T. 2004. Variations in tree community composition and structure in a fragment of tropical semideciduous forest in southeastern Brazil related to different human disturbance histories. Forest Ecology and Management 198:319-339.

UDULUTSCH, R.G., ASSIS, M.A. \& PICCHI, D.G. 2004. Florística de trepadeiras numa floresta estaciomal semidecídua, Rio Claro - Araras, Estado de São Paulo, Brasil. Revista Brasileira de Botânica 27:125-134.

VELOSO, H.P. 1991. Manual técnico da vegetação brasileira. IBGE, Rio de Janeiro.

WRIGHT, D.D., JESSEN, J.H., BURKE, P. \& GÓMEZ DE SILVA-GARZA, H. 1997. Tree and liana enumeration and diversity on a one-hectare plot in Papua New Guinea. Biotropica 29:250-260. 